\title{
A CHARACTERIZATION OF EXTREMAL ELEMENTS IN SOME LINEAR PROBLEMS ${ }^{1}$
}

\author{
Vitalii V. Arestov \\ Krasovskii Institute of Mathematics and Mechanics, \\ Ural Branch of the Russian Academy of Sciences and \\ Ural Federal University, Ekaterinburg, Russia \\ vitalii.arestov@urfu.ru
}

\begin{abstract}
We give a characterization of elements of a subspace of a complex Banach space with the property that the norm of a bounded linear functional on the subspace is attained at those elements. In particular, we discuss properties of polynomials that are extremal in sharp pointwise Nikol'skii inequalities for algebraic polynomials in a weighted $L_{q}$-space on a finite or infinite interval.
\end{abstract}

Key words: Complex Banach space, Bounded linear functional on a subspace, Algebraic polynomial, Pointwise Nikol'skii inequality.

\section{Bounded linear functionals in complex Banach spaces}

\subsection{Introduction. Statement of the problem}

Let $X=X_{\mathbb{C}}$ be a complex Banach space (more precisely, a Banach space over the field $\mathbb{C}$ of complex numbers), let $S(X)$ be its unit sphere, and let $X^{*}=X_{\mathbb{C}}^{*}$ be the dual space of $X$, i.e., the space of complex-valued bounded linear (over the field $\mathbb{C}$ of complex numbers) functionals $F$ on $X$ with the norm

$$
\|F\|_{X^{*}}=\sup \left\{|F(x)|: x \in X,\|x\|_{X}=1\right\} .
$$

Let $P$ be a (closed) subspace of $X$, and let $\psi$ be a bounded linear functional on $P$. We denote by

$$
D(\psi ; P)=\sup \left\{|\psi(p)|: p \in P,\|p\|_{X}=1\right\}
$$

the norm of the functional $\psi$ on the subspace $P$. In what follows, we assume that $\psi \not \equiv 0$, so that $D(\psi ; P)>0$. The value $D(\psi ; P)$ is the smallest possible (the best) constant in the inequality

$$
|\psi(p)| \leq D(\psi ; P)\|p\|_{X}, \quad p \in P .
$$

Nonzero elements $p$ of the subspace $P$ with the property that inequality (1.2) turns into an equality for them (if such elements exist) will be called extremal elements in this inequality. Elements $p$ of the unit sphere $S(P)=S(X) \cap P$ of the subspace $P$ that solve problem (1.1), i.e., those with the property that the supremum in (1.1) is attained at $p$, will be called extremal elements in problem (1.1). We will use the same terminology also in other similar situations. It is clear that an element $\varrho \in P$ is extremal in inequality (1.2) if and only if the element $\varrho /\|\varrho\|_{X}$ is extremal in problem (1.1). In this sense, extremal elements in problem (1.1) and inequality (1.2) coincide. The aim of this papers is exactly to characterize extremal elements in inequality (1.2) or in problem (1.1), which is the same.

\footnotetext{
${ }^{1}$ This work was supported by the Program of the Ural Branch of the Russian Academy of Sciences (project no. $15-16-1-4)$
} 
On the set

$$
P[1](\psi)=\{p \in P: \psi(p)=1\}
$$

of elements of $P$ where the functional $\psi$ takes the value 1 , we consider the value

$$
\Delta(\psi ; P)=\inf \left\{\|p\|_{X}: p \in P[1](\psi)\right\}
$$

which is the least deviation of class (1.3) from zero in $X$. It is clear that $\Delta(\psi ; P)=1 / D(\psi ; P)$. Moreover, extremal elements in problem (1.4) and inequality (1.2) coincide. More precisely, each extremal element of problem (1.4) is extremal in (1.2); conversely, if $\varrho$ is an extremal element of inequality (1.2), then $\varrho / \psi(\varrho)$ is extremal in (1.4). Thus, determining the sharp constant in inequality (1.2) is equivalent to determining the least deviation (1.4) of class (1.3) from zero.

Value (1.4) can be interpreted as the best approximation of an arbitrary element $\rho \in P[1](\psi)$ in the space $X$ by the annihilator

$$
P(\psi)=P[0](\psi)=\{p \in P: \psi(p)=0\}
$$

of the functional $\psi$ in $P$, namely,

$$
\Delta(\psi ; P)=\inf \left\{\|\rho-p\|_{X}: p \in P(\psi)\right\} .
$$

There is a rich theory developed to study problems of type (1.4) in real Banach spaces. This theory is based on arguments of duality; see, e.g., [13, Ch. 2]. In order to use this approach in the complex case, however, one needs in addition to discuss some questions of geometry of complex spaces.

In papers [1-4] coauthored by the author of the present paper, the authors studied the Nikol'skii inequality between the uniform norm of a polynomial and its norm in the space $L_{q}^{v}=L_{q}^{v}(\mathbb{I})$ with a weight $v$ and $1 \leq q<\infty$ on the set of algebraic polynomials $\mathscr{P}_{n}$ of degree at most $n \geq 1$ on a finite or infinite interval $\mathbb{I}$. One of the steps in these investigations was the study of the sharp inequality

$$
\left|p_{n}\left(z_{0}\right)\right| \leq D\left\|p_{n}\right\|_{L_{q}^{v}}, \quad p_{n} \in \mathscr{P}_{n},
$$

for an end point $z_{0}$ of the interval $\mathbb{I}$. Inequality (1.7) is a special case of (1.2) for $X=L_{q}^{v}(\mathbb{I})$, $P=\mathscr{P}_{n}$, and $\psi\left(p_{n}\right)=p_{n}\left(z_{0}\right)$. Results of [1-4] related to inequality (1.7) motivated the author to consider problem (1.2).

\subsection{Main result}

We will study problem (1.1) under the following two assumptions.

(R) Assume that the norm of any bounded linear functional $\phi$ on $P$, i.e., $\phi \in P^{*}$, is attained at some point $p=p(\phi) \in P$.

According to James' theorem [11] (see also [10, p. 643], [17, Ch. 1, Sect. 2, Corollary 2.4]), this property is equivalent to the reflexivity of the space $P$. Note that property $(\mathrm{R})$ is fulfilled if the subspace $P$ is finite-dimensional.

If a functional $F \in X^{*}, F \neq 0$, attains its norm at an element $x \in X, x \neq 0$, and if $F(x)>0$, or-which is the same in this case - if

$$
F(x)=\|F\|_{X^{*}}\|x\|_{X},
$$

we will say that the functional $F$ possesses the $N$-property at the element $x$, or, shortly, the $N[x]$ property. By the complex variant of the Hahn-Banach Theorem (cf. [8, Ch. II, Sect. 3, Theorem 11] 
or [12, Ch. III, Sect. 5.4]), a functional with this property always exists. However, it may be not unique. In a complex Banach space, a functional $F \in X^{*}$ is called a supporting functional at a point $x$ (or, more precisely, a supporting or a tangent functional at a point $x$ to the sphere $S_{\|x\|}(X)$ of radius $\|x\|$ with center at 0 ), if its real part $f=\operatorname{Re} F$ is a real supporting (tangent) functional, see, e.g., [8, Ch. V, Sect. 9.4]. Indeed, for a functional $F \in X^{*}$ in a complex Banach space, the properties that the functional possesses the $N$-property at a point $x$ and that its real part is a supporting functional are equivalent; we will discuss this below in Section 1.3. Starting from this point, we will interpret the $N[x]$-property of a functional $F \in X^{*}$ as a property of the functional $F \in X^{*}$ to be a supporting functional at the point $x$.

A point $x \in S(X)$ is called a smooth point of the sphere $S(X)$ if there exists only one supporting functional at $x$. If every point of the unit sphere of a space is a smooth point, then the space is called smooth. For details concerning smooth points of the unit sphere and, in general, of convex closed sets in real Banach spaces see, e.g., [7, Ch. I, Sect. 2, Theorems 1, 2] and [6, Ch. VII, Sect. 2]. The smoothness in complex Banach spaces has some special features; it will be discusses in Section 1.3 below.

The second assumption is the following one.

(Г) Assume that all points of the unit sphere $S(P)=S(X) \cap P$ of the subspace $P$ are smooth points of the unit sphere $S(X)$ of the space $X$.

Taking into account that problem (1.1) has the interpretation (1.4) in terms of approximations, one may expect the following result.

Theorem 1. Assume that a Banach space $X$ and its subspace $P$ satisfy properties $(\mathrm{R})$ and $(\Gamma)$. Then the norm of a bounded linear functional $\psi$ on $P$ is attained at an element $\varrho \in S(P)$ if and only if the supporting functional $F=F[\varrho] \in X^{*}$ of the element $\varrho \in P$ vanishes on the set (1.5), i.e.,

$$
F[\varrho](s)=0 \quad \text { for all } \quad s \in P(\psi) \text {. }
$$

Under the assumptions of the theorem, an extremal element with the property that the norm of the functional $\psi$ on $P$ is attained at it always exists but it is not necessarily unique; see the example after the proof of Theorem 4 in Section 2.2. To ensure the uniqueness of the extremal element, one needs additional restrictions on the problem. For example, if the space $X$ is strictly normed then the extremal element is unique for every (bounded linear) functional on every subspace.

In the first section of the present paper, Theorem 1 will be proved and discussed. In the second section, Theorem 1 will be applied to obtain a corresponding statement for the pointwise inequality

$$
\left|p_{n}(z)\right| \leq D(z)\left\|p_{n}\right\|_{L_{q}^{v}(\mathbb{I})}, \quad p_{n} \in \mathscr{P}_{n},
$$

where $z \in \mathbb{I}$. In papers [1-4], extremal polynomials of inequality (1.7) (in the case when $z$ is an end point of an interval) were characterized in terms that are formally different from those of Theorem 1. We will show in Section 2.2 that, in fact, Theorem 2 from [3] and its analogs from $[1,2,4]$ follow from Theorem 1 .

We will prove Theorem 1 using the natural arguments in terms of duality. However, the fact that $X$ is a complex Banach space causes additional difficulties. In particular, one needs to first discuss the smoothness property for points of the unit sphere $S(X)$ of a (complex) Banach space $X$.

\subsection{Smoothness in complex Banach spaces}

In real Banach spaces, a smooth point of the unit sphere can be for example characterized by the fact that the norm of the space is Gateaux differentiable at this point. For a real Banach space, 
the strict convexity of the dual space is a sufficient condition for the smoothness of the original space. The inverse statement does not hold in the general case. The smoothness of a space implies the strict convexity of the dual space only for reflexive spaces. Details on these topics can be found, e.g., in [7, Ch. I, Sect. 2, Theorems 1, 2] and [6, Ch. VII, Sect. 2]. In this section, we discuss smoothness in complex Banach spaces. The author neither claims that the results are novel nor that the ideas are original.

Let $X=X_{\mathbb{C}}$ be a complex Banach space. We also may consider this space as a real Banach space $X=X_{\mathbb{R}}$, i.e., a linear space over the field $\mathbb{R}$ of real numbers. Let $X_{\mathbb{R}}^{*}$ be the corresponding dual (real) Banach space, i.e., the space of real-valued bounded linear (over the field $\mathbb{R}$ of real numbers) functionals on $X_{\mathbb{R}}$.

The following statement is not new, cf. [8, Ch. II, Sect. 3, Theorem 11] or [9, Ch. 10, Sect. 1, Lemma 1.1]. We will give it here in the form we need in what follows. Moreover, it is useful for our purposes to give a proof of this statement.

Lemma 1. The formula

$$
F(x)=f(x)-i f(i x), \quad x \in X,
$$

where $F \in X_{\mathbb{C}}^{*}$ and $f \in X_{\mathbb{R}}^{*}$, sets a one-to-one correspondence between the spaces $X_{\mathbb{C}}^{*}$ and $X_{\mathbb{R}}^{*}$. Moreover, mapping (1.10) is an isometry, i.e.,

$$
\|F\|_{X_{\mathbb{C}}^{*}}=\|f\|_{X_{\mathbb{R}}^{*}}
$$

P r o o f. For a complex functional $F \in X_{\mathbb{C}}^{*}$, we consider its real part $f=\operatorname{Re} F$; it is a functional from $X_{\mathbb{R}}^{*}$. The functional $F$ is uniquely determined by $f=\operatorname{Re} F$ by means of formula (1.10). Indeed, define $g=-\operatorname{Im} F$, then $F(x)=f(x)-i g(x), x \in X$. By the (complex) homogeneity of the functional $F$, we have $F(x)=-i F(i x)=-i f(i x)+g(i x), x \in X$. Consequently, $g(x)=f(i x)$, which proves representation (1.10).

Conversely, let $f \in X_{\mathbb{R}}^{*}$. Consider a (complex) functional $F$ given by formula (1.10). Obviously, $F$ is additive. Next we will show that it is (complex) homogeneous. For a point $x \in X$ and a number $\zeta=\alpha+i \beta \in \mathbb{C}$, we have

$$
\begin{gathered}
F(\zeta x)=F((\alpha+i \beta) x)=f((\alpha+i \beta) x)-i f(i(\alpha+i \beta) x)= \\
=\alpha f(x)+\beta f(i x)-i \alpha f(i x)+i \beta f(x)=(\alpha+i \beta) f(x)+(\beta-i \alpha) f(i x)= \\
=(\alpha+i \beta)(f(x)-i f(i x))=\zeta F(x) .
\end{gathered}
$$

Thus, we see that functional (1.10) is homogeneous.

It follows that formula (1.10) sets a one-to-one correspondence between the complex and the real dual spaces $X_{\mathbb{C}}^{*}$ and $X_{\mathbb{R}}^{*}$, respectively.

Now we show that (1.10) is an isometry, i.e., property (1.11) holds. The inequality $\|f\|_{X_{\mathbb{R}}^{*}} \leq$ $\|F\|_{X_{\mathbb{C}}^{*}}$ is obvious. Further on, for an arbitrary point $x \in X$ and real $\theta$, we have

$$
e^{i \theta} F(x)=F\left(e^{i \theta} x\right)=f\left(e^{i \theta} x\right)-i f\left(i e^{i \theta} x\right) .
$$

In particular, for $\theta=-\arg (F(x))$, the latter equality takes the form

$$
|F(x)|=f\left(e^{i \theta} x\right)-i f\left(i e^{i \theta} x\right)=f\left(e^{i \theta} x\right) .
$$

Consequently, $|F(x)| \leq\|f\|_{X_{\mathbb{R}}^{*}}\|x\|, x \in X$, and therefore the estimate $\|F\|_{X_{\mathbb{C}}^{*}} \leq\|f\|_{X_{\mathbb{R}}^{*}}$ holds. Thus, (1.11) holds. This proves the lemma.

All further statements in this section are in fact consequences of Lemma 1. 
Lemma 2. A complex functional $F \in X_{\mathbb{C}}^{*}$ attains its norm at a point $x \in S(X)$ and $F(x)>0$ if and only if its real part $f=\operatorname{Re} F$ has the same properties.

P r o o f. Suppose $F$ is as described in the lemma. By (1.8) and (1.11), we have

$$
\|F\|_{X_{\mathbb{C}}^{*}}=\|f\|_{X_{\mathbb{R}}^{*}}=F(x)=f(x) .
$$

Consequently, $f$ has the same properties as $F$. Conversely, suppose $f$ has the described properties. Then, by (1.11), we have

$$
f(x) \leq \sqrt{(f(x))^{2}+(f(i x))^{2}}=|F(x)| \leq\|F\|_{X_{\mathbb{C}}^{*}}=\|f\|_{X_{\mathbb{R}}^{*}} .
$$

Consequently, $f(x)=F(x)=\|F\|_{X_{\mathbb{C}}^{*}}$; hence, $F(x)>0$. Thus, $F$ has the described properties, too.

As we have mentioned above, a functional $F \in X^{*}$ in a complex Banach space is called a supporting functional at a point $x$ (to the sphere $S_{\|x\|}(X)$ of radius $\|x\|$ with center at 0 ) if its real part $\operatorname{Re} F$ is a (real) supporting functional, cf. [8, Ch. V, Sect. 9.4]. Due to Lemma 2, the $N$-property of the functional $F \in X^{*}$ at a point $x$ is equivalent to the property that $F \in X^{*}$ is a supporting functional at this point.

Theorem 2. Assume that the space $X^{*}=X_{\mathbb{C}}^{*}$ of complex bounded linear functionals in a complex Banach space $X$ is strictly convex. Then $X$ is smooth.

P r o o f. Recall that a Banach space is called strongly convex if its unit sphere does not contain any non-degenerate segments, see, e.g. [8, Ch. V, Sect. 11.7]. As we have mentioned above, the statement of the theorem is well-known for real Banach spaces, cf. [7, Ch. I, Sect. 2, Theorems 1 and 2], [6, Ch. VII, Sect. 2].

Using Lemma 1, it is not difficult to see that $X_{\mathbb{C}}^{*}$ is strongly convex if and only if $X_{\mathbb{R}}^{*}$ is. Thus, under the assumptions of the theorem, the space $X_{\mathbb{R}}$ is smooth. This means that, at any point $x \in S(X)$, there is only one real bounded linear functional $f$ whose norm is equal to 1 and is attained at $x$, with $f(x)>0$. By Lemma 1, this implies that, at every point $x \in S(X)$, there is only one functional $F \in X_{\mathbb{C}}^{*}$ with the unit norm and with the $N$-property at the point $x$. But this means that the space $X=X_{\mathbb{C}}$ is smooth.

\subsection{Proof of Theorem 1}

Theorem 1 follows from the two auxiliary statements proved below. In what follows, we will suppose without loss of generality that all supporting functionals $F=F[x]$ at points $x \in X, x \neq 0$, have the norm $\|F\|_{X^{*}}=1$.

\subsubsection{Auxiliary statements}

Lemma 3. Assume that a Banach space $X$ and its subspace $P$ satisfy properties $(\mathrm{R})$ and $(\Gamma)$. Let $\varrho \in P, \varrho \not \equiv 0$, be an extremal element of problem (1.1), and let $F=F[\varrho] \in S\left(X^{*}\right)$ be the supporting functional at the element $\varrho \in P$. Then the following representation holds:

$$
\psi(p)=\gamma(P) F[\varrho](p), \quad p \in P,
$$

where $\gamma(P)$ is a constant with the property $|\gamma(P)|=D(\psi, P)$. 
P r o o f. The functional $\psi$ is a bounded linear functional on the space $P$ endowed with the norm $\|\cdot\|_{X}$, and the norm of this functional on $P$ is equal to (1.1). By the Hahn-Banach theorem (cf. [8, Ch. II, Sect. 3, Theorem 11] or [12, Ch. III, Sect. 5.4]), the functional $\psi$ can be extended to a functional on the whole space $X$ with the same norm; we denote this extension by $\Psi$.

Since the functional $\Psi$ is an extension of the functional $\psi$ from $P$ to $X$ with the same norm, the norm of the functional $\Psi$ in the space $X$ is attained at an extremal element $\varrho \in P$ of problem (1.1). By property $(\Gamma)$, the functional $\Psi$ differs from the functional $F=F[\varrho] \in X^{*}$ only by a constant factor $\gamma(P)$ :

$$
\Psi(p)=\gamma(P) F[\varrho](p), \quad p \in X .
$$

In particular, (1.12) holds. Taking $p=\varrho$ in (1.12), we see that $|\gamma(P)|=D(\psi, P)$. This proves representation (1.12).

Lemma 4. Assume that a Banach space $X$ and its subspace $P$ satisfy properties $(\mathrm{R})$ and $(\Gamma)$. If an element $\varrho \in P, \varrho \neq \equiv 0$, or, more precisely, the supporting functional $F=F[\varrho] \in S\left(X^{*}\right)$ at the element $\varrho$ has property (1.9), then $\varrho$ is an extremal element of problem (1.1).

P r o o f. Assume that an element $\varrho \in P, \varrho \neq \equiv 0$, has property (1.9); without loss of generality, we may assume that $\|\varrho\|_{X}=1$. We consider the linear functional on the set $P$ defined by the formula

$$
\Psi_{0}(p)=F[\varrho](p) .
$$

For any $p \in P$, the element $s=\psi(\varrho) p-\psi(p) \varrho$ belongs to the set $P(\psi)$. Due to (1.9), we have

$$
\psi(\varrho) \Psi_{0}(p)-\psi(p) \Psi_{0}(\varrho)=0 .
$$

By (1.8) and (1.13), we have $\Psi_{0}(\varrho)=F[\varrho](p)=1$. Thus, (1.14) can be rewritten as

$$
\psi(p)=\psi(\varrho) \Psi_{0}(p), \quad p \in P .
$$

We conclude that

$$
|\psi(p)|=|\psi(\varrho)|\left|\Psi_{0}(p)\right| \leq|\psi(\varrho)|\|p\| .
$$

Consequently, $D(\psi, P) \leq|\psi(\varrho)|$. Since $\|\varrho\|_{X}=1$, we have $D(\psi, P) \geq|\psi(\varrho)|$. It follows that $D(\psi, P)=|\psi(\varrho)|$ and the element $\varrho$ is extremal in problem (1.1).

\subsubsection{Proof of Theorem 1}

Formula (1.12) implies that an extremal element of problem (1.1) has property (1.9). According to Lemma 4, the inverse statement holds. This proves Theorem 1.

\section{Bounded linear functionals on the set of algebraic polynomials in spaces $L_{q}^{v}, 1 \leq q<\infty$}

Assume that $\mathbb{I}$ is a finite or infinite closed interval of the real line and $v$ is a nonnegative function that is integrable and almost everywhere nonzero on $\mathbb{I}$; we will call such functions weights on $\mathbb{I}$. Denote by $L_{q}=L_{q}^{v}(\mathbb{I}), 1 \leq q<\infty$, the space of (complex-valued) measurable functions $f$ on $\mathbb{I}$ such that the product $|f|^{q} v$ is integrable on $\mathbb{I}$; this is a Banach space with the norm

$$
\|f\|_{L_{q}^{v}(\mathbb{I})}=\left(\int_{\mathbb{I}}|f(x)|^{q} v(x) d x\right)^{1 / q}, \quad f \in L_{q}^{v}(\mathbb{I}) .
$$


For $q=\infty$, we assume that $L_{\infty}^{v}(\mathbb{I})$ is the space $L_{\infty}=L_{\infty}(\mathbb{I})$ of essentially bounded functions on $\mathbb{I}$ with the norm

$$
\|f\|_{L_{\infty}(\mathbb{I})}=\operatorname{ess} \sup \{|f(t)|: t \in \mathbb{I}\} .
$$

Let $\mathscr{P}_{n}=\mathscr{P}_{n}(\mathbb{C})$ for $n \geq 0$ be the set of algebraic polynomials (in one variable) of degree at most $n$ with complex coefficients. We will assume that $\mathscr{P}_{n} \subset L_{q}^{v}(\mathbb{I})$; this condition is equivalent to the fact that the function $1+|x|^{n}$ belongs to the space $L_{q}^{v}(\mathbb{I})$.

\subsection{Arbitrary bounded linear functionals on the space of algebraic polyno- mials}

Assume that $\psi$ is a linear functional on $\mathscr{P}_{n}$. Since $\mathscr{P}_{n}$ is finite-dimensional, the functional $\psi$ on $\mathscr{P}_{n}$ is bounded and its norm

$$
D\left(\psi ; \mathscr{P}_{n}\right)_{q}=\sup \left\{|\psi(p)|: p \in \mathscr{P}_{n},\|p\|_{L_{q}^{v}(\mathbb{I})}=1\right\}
$$

is attained at a certain polynomial $\varrho_{n}=\varrho_{\psi, \mathscr{P}_{n}, q} \in \mathscr{P}_{n}$ with the property

$$
\left\|\varrho_{\psi, \mathscr{P}_{n}, q}\right\|_{\left.L_{q} \mathbb{I}, v\right)}=1 .
$$

In the study of extremal problems for polynomials, it is an important fact that the value $D_{n}(\psi)=$ $D\left(\psi ; \mathscr{P}_{n}\right)_{q}$ is the smallest possible (the best) constant in the inequality

$$
|\psi(p)| \leq D_{n}(\psi)\|p\|_{L_{q}^{v}(\mathbb{I})}, \quad p \in \mathscr{P}_{n} .
$$

Inequality (2.2) turns into an equality at the polynomial $\varrho_{n}$, i.e., $\varrho_{n}$ is extremal in (2.2). It is clear that the polynomial $c \varrho_{n}$ with an arbitrary constant $c \in \mathbb{C}$ is also extremal in (2.2). If all extremal polynomials in inequality $(2.2)$ have the form $c \varrho_{n}, c \in \mathbb{C}$, we say that $\varrho_{n}$ is the unique extremal polynomial of inequality (2.2) (or of problem (2.1)). In what follows, we assume that $\psi \neq \equiv 0$; this is equivalent to the fact that $\left|\psi\left(\varrho_{n}\right)\right|=D\left(\psi ; \mathscr{P}_{n}\right)>0$.

Consider the annihilator

$$
\mathscr{P}_{n}(\psi)=\left\{p \in \mathscr{P}_{n}: \psi(p)=0\right\}
$$

of the functional $\psi$ in the set $\mathscr{P}_{n}$. This set is a subspace of $\mathscr{P}_{n}$ of codimension 1 . This subspace is formed by polynomials of the form

$$
s=p-\frac{\psi(p)}{\psi\left(\varrho_{n}\right)} \varrho_{n}, \quad p \in \mathscr{P}_{n} .
$$

Theorem 3. Let $1 \leq q<\infty$. A polynomial $\varrho_{n}=\varrho_{\psi, \mathscr{P}_{n}, q} \in \mathscr{P}_{n}$ which is extremal in inequality (2.2) exists. A polynomial $\varrho_{n} \in \mathscr{P}_{n}$ is extremal if and only if

$$
\int_{\mathbb{I}} s(x) v(x)\left|\varrho_{n}(x)\right|^{q-1} \operatorname{sign} \varrho_{n}(x) d x=0 \quad \text { for all } \quad s \in \mathscr{P}_{n}(\psi) .
$$

In the case when $1<q<\infty$, this extremal polynomial is unique (up to a constant factor).

P r o o f. We check that all assumptions of Theorem 1 are fulfilled under the assumptions of Theorem 3. The set $\mathscr{P}_{n}=\mathscr{P}_{n}(\mathbb{C})$ of algebraic polynomials of degree at most $n$ is a finitedimensional subspace of $L_{q}^{v}(\mathbb{I})$. This guarantees that property (R) holds.

Now let us verify property $(\Gamma)$. We start with the case $q=1$. The dual space of $L=L_{1}^{v}(\mathbb{I})$ is the space $L_{\infty}=L_{\infty}(\mathbb{I})$ of essentially bounded functions on $\mathbb{I}$. A functional $\Phi \in X^{*}$ has the representation

$$
\Phi(f)=\int_{\mathbb{I}} f(t) \bar{\phi}(t) v(t) d t, \quad f \in L_{1}^{v}(\mathbb{I}),
$$

where $\phi \in L_{\infty}(\mathbb{I})$ and $\|\Phi\|_{L^{*}}=\|\phi\|_{L_{\infty}(\mathbb{I})}$. 
For a pair of functions $\phi \in L_{\infty}(\mathbb{I})$ and $f \in L_{1}^{v}(\mathbb{I})$, the inequality

$$
\left|\int_{\mathbb{I}} f(t) \bar{\phi}(t) v(t) d t\right| \leq\|\phi\|_{L_{\infty}(\mathbb{I})}\|f\|_{L_{1}^{v}(\mathbb{I})}
$$

turns into an equality if and only if the following three conditions hold:

(1) the set

$$
\mathbb{I}(\phi)=\left\{t \in \mathbb{I}:|\phi(t)|=\|\phi\|_{L_{\infty}(\mathbb{I})}\right\}
$$

where the absolute value of the function $\phi$ takes its maximum has a positive measure;

(2) the function $f$ vanishes almost everywhere outside the set $\mathbb{I}(\phi)$;

(3) the product $f \bar{\phi}$ has the same sign almost everywhere on the set

$$
\Theta f=\{t \in \mathbb{I}: f(t) \neq 0\} .
$$

Taking into account these observations, it is not difficult to conclude that a supporting functional of a function $f \in S\left(L_{1}^{v}(\mathbb{I})\right)$ has the form (2.5), where the function $\phi$ satisfies the following conditions: $\phi=\operatorname{sign} f$ almost everywhere on $\Theta f$ and $|\phi| \leq 1$ almost everywhere outside $\Theta f$.

Consequently, a function $f \in S\left(L_{1}^{v}(\mathbb{I})\right)$ is a smooth point of the unit sphere of the space $L_{1}^{v}(\mathbb{I})$ if and only if $f$ is nonzero almost everywhere on $\mathbb{I}$; the supporting functional in this case has the form (2.5) with the function $\phi=\operatorname{sign} f$. In particular, this property holds in the case if $f$ is an algebraic polynomial. Thus, under the assumptions of Theorem 3 for $q=1$, property $(\Gamma)$ holds.

For $1<q<\infty$, the dual space of $L_{q}=L_{q}^{v}(\mathbb{I})$ is $L_{q^{\prime}}=L_{q^{\prime}}^{v}(\mathbb{I}), 1 / q+1 / q^{\prime}=1$. The space $L_{q^{\prime}}^{v}(\mathbb{I})$ with $1<q^{\prime}<\infty$ is uniformly convex; hence, the space $L_{q}^{v}(\mathbb{I})$ is smooth.

Thus, we have shown that all assumptions of Theorem 1 are fulfilled under the assumptions of Theorem 3. Thus, also the statement of Theorem 1 holds. For $1<q<\infty$, the space $L_{q}^{v}(\mathbb{I})$ is uniformly smooth, hence, the extremal polynomial in inequality $(2.2)$ is unique. This proves the theorem.

\subsection{Pointwise Nikol'skii inequality for algebraic polynomials on an interval}

Let $u$ be another, this time continuous weight on $\mathbb{I}$. Along with $L_{q}^{v}(\mathbb{I})$, we consider the space $C=C(\mathbb{I}, u)$ of complex-valued continuous functions $f$ such that the product $f u$ is bounded on $\mathbb{I}$, endowed the (uniform weighted) norm

$$
\|f\|_{C(\mathbb{I}, u)}=\sup \{|f(x) u(x)|: x \in \mathbb{I}\} .
$$

We will assume that $\mathscr{P}_{n}$ is contained not only in $L_{q}^{v}(\mathbb{I})$ but also in $C(\mathbb{I}, u)$; the latter is equivalent to the fact that the function $u(x)\left(1+|x|^{n}\right)$ is bounded on $\mathbb{I}$.

Denote by $M(n)=M(n, u, v)_{q}$ the best (the smallest possible) constant in the inequality

$$
\|p\|_{C(\mathbb{I}, u)} \leq M(n)\|p\|_{L_{q}^{v}(\mathbb{I})}, \quad p \in \mathscr{P}_{n},
$$

on the set $\mathscr{P}_{n}$. Inequality (2.6) is a special case of an inequality between different metrics, or the Nikol'skii inequality. Such inequalities appeared for the first time in Nikol'skii's paper [15] and, shortly after that, in a paper by Szegő and Zygmund [18]. Similar inequalities and, more generally, inequalities between the uniform norm and weighted integral norms of algebraic and trigonometric polynomials and their derivatives have been studied over a period of more than 150 years, starting with the works of Chebyshev and his students - the Markov brothers. Further information and references on this topic can be found, e.g., in monographs [5, 14] and papers [2, 3, 16].

Along with (2.6), we consider the pointwise inequality

$$
\left|p_{n}(z)\right| \leq D_{n}[z]\left\|p_{n}\right\|_{L_{q}^{v}(\mathbb{I})}, \quad p_{n} \in \mathscr{P}_{n},
$$


with the smallest possible constant $D_{n}[z]=D(n, v, q ; z)$ for points $z \in \mathbb{I}$. Such inequalities are of independent interest, but they are also important in connection with inequality (2.6) since

$$
M(n)=\sup \left\{D_{n}[z] u(z): z \in \mathbb{I}\right\} .
$$

In a number of important cases, the product $D_{n}[z] u(z)$ takes its maximal value with respect to $z \in \mathbb{I}$ at an end point of the interval $\mathbb{I}$; see, e.g., $[2-4,16]$ and the references therein.

In the setup we consider in this section, (1.6) and (1.3) take the form

$$
\begin{gathered}
\Delta_{n}[z]=\inf \left\{\left\|p_{n}\right\|_{L_{q}^{v}(\mathbb{I})}: p_{n} \in \mathscr{P}_{n}[z]\right\}, \\
\mathscr{P}_{n}[z]=\left\{p_{n} \in \mathscr{P}_{n}: p_{n}(z)=1\right\} .
\end{gathered}
$$

Theorem 4. For $1 \leq q<\infty$, the following is true for an extremal polynomial in inequality (2.7).

(1) An extremal polynomial $\varrho_{n}$ in inequality (2.7) exists, it has real coefficients, all its roots are real, and its degree is at least $n-1$. In the case when $1<q<\infty$, the extremal polynomial is unique.

(2) A polynomial $\varrho_{n} \in \mathscr{P}_{n}$ is extremal in inequality (2.7) if and only if

$$
\int_{\mathbb{I}} p_{n-1}(x)(x-z) v(x)\left|\varrho_{n}(x)\right|^{q-1} \operatorname{sign} \varrho_{n}(x) d x=0 \quad \text { for all } \quad p_{n-1} \in \mathscr{P}_{n-1} .
$$

P r o o f. Inequality (2.7) is a special case of inequality (2.2) for the functional $\psi(p)=p(z)$, $p \in \mathscr{P}_{n}$. In this case, set (2.3) is formed by polynomials of the form $s(x)=(x-z) p_{n-1}(x)$, $p_{n-1} \in \mathscr{P}_{n-1}$. Therefore, condition (2.4) for an extremal polynomial $\varrho_{n}$ in inequality (2.7) takes the form (2.9). Thus, the second statement of Theorem 4 is proved. Without loss of generality, we may assume that $\varrho_{n}(z)=1$; for, consider $\varrho_{n} / \varrho_{n}(z)$ instead of the polynomial $\varrho_{n}$, if necessary.

The polynomial $\varrho_{n}$ is also a solution of problem (2.8). We will study some properties of the polynomial $\varrho_{n}$ using this fact. In general, the coefficients $\left\{c_{k}\right\}_{k=0}^{n}$ of the polynomial $\varrho_{n}$ are complex, namely, $c_{k}=a_{k}+i b_{k}, a_{k}, b_{k} \in \mathbb{R}$. We write $\varrho_{n}$ in the form $\varrho_{n}=u_{n}+i v_{n}$, where

$$
u_{n}(x)=\left(\operatorname{Re} \varrho_{n}\right)(x)=\sum_{k=0}^{n} a_{k} x^{k}, \quad v_{n}(x)=\left(\operatorname{Im} \varrho_{n}\right)(x)=\sum_{k=0}^{n} b_{k} x^{k}
$$

are real polynomials (on $\mathbb{R}$ ). Obviously, $u_{n}(z)=\varrho_{n}(z)=1$; hence, $u_{n} \in \mathscr{P}_{n}[z]$. If $b_{k} \neq 0$ for at least one $k, 0 \leq k \leq n$, then the strict inequality $\left|u_{n}(x)\right|<\left|\varrho_{n}(x)\right|$ holds for all $x \in \mathbb{I}$ except for zeros of the polynomial $v_{n}$. Consequently, the strict inequality $\left\|u_{n}\right\|_{L_{q}^{v}(\mathbb{I})}<\left\|\varrho_{n_{n}}\right\|_{L_{q}^{v}(\mathbb{I})}$ holds for the norms of these polynomials. The latter is a contradiction to the fact that the polynomial $\varrho_{n}$ is extremal in (2.8). This proves that the coefficients of the polynomial $\varrho_{n}$ are real.

Assume that the polynomial $\varrho_{n}$ has a zero $\zeta$ which is not real. Since the polynomial $\varrho_{n}$ is real, we conclude that $\bar{\zeta}$ is also a zero of $\varrho_{n}$. Consequently, $\varrho_{n}(x)=q_{n-2}(x)|x-\zeta|^{2}$, where $q_{n-2}$ is a polynomial of degree at most $n-2$. The polynomial $p_{n-1}(x)=q_{n-2}(x)(x-z)$ has degree at most $n-1$. The left-hand side of $(2.9)$ is positive for this polynomial:

$$
\begin{gathered}
\int_{\mathbb{I}} p_{n-1}(x)(x-z) v(x)\left|\varrho_{n}(x)\right|^{q-1} \operatorname{sign} \varrho_{n}(x) d x= \\
=\int_{\mathbb{I}}(x-z)^{2} v(x)\left|q_{n-2}(x)\right|^{q}|x-\zeta|^{2(q-1)} \operatorname{sign} q_{n-2}(x) d x>0 .
\end{gathered}
$$

This contradicts property (2.9). Thus, the polynomial $\varrho_{n}$ can have only real zeros. 
Finally, let us check that the exact degree of the polynomial $\varrho_{n}$ is $n$ or $n-1$. Indeed, if $\varrho_{n}$ has degree at most $n-2$, then the polynomial $p_{n-1}(x)=(x-z) \varrho_{n}(x)$ has degree at most $n-1$. The integral on the left-hand side of (2.9) is positive for this polynomial. This contradicts property (2.9). The theorem is proved.

Example. Consider the special case of problem (2.7) in the space $L=L(-1,1)$ of functions that are integrable over the interval $I=[-1,1]$ with the unit weight, with $n=1$ and $z=0$. In other words, we are interested in the sharp inequality

$$
|p(0)| \leq D\|p\|_{L}, \quad p \in \mathscr{P}_{1} .
$$

It is easy to verify that we have the formula

$$
p(0)=\frac{1}{2} \int_{-1}^{1} p(t) d t, \quad p \in \mathscr{P}_{1} .
$$

Using this formula, it is straightforward that the best constant in inequality (2.10) is $D=1 / 2$ and that every polynomial of constant sign on $(-1,1)$ is extremal. Thus, an extremal polynomial in inequality (2.7) may be not unique, may have (real) zeros outside the interval $\mathbb{I}$, and may have the exact degree $n-1$.

For an end point $z$ of the interval $\mathbb{I}$, we are able to derive more information about the properties of extremal polynomials in inequality (2.7) from Theorem 4. In this case, the product $(x-z) v(x)$ on the left-hand side of (2.9) has constant sign on $\mathbb{I}$. Therefore, using property (2.9), it is not difficult to see that an extremal polynomial $\varrho_{n}$ has degree exactly $n$, all $n$ zeros of this polynomial are simple and lie in the interior of the interval $\mathbb{I}$. Property (2.9) implies also that the extremal polynomial $\varrho_{n}$ is unique for all $1 \leq q<\infty$. Indeed, let $\varrho_{n}$ and $\eta_{n}$ be two polynomials that solve problem (2.8). The same property is true for their half-sum $\left(\varrho_{n}+\eta_{n}\right) / 2$; therefore, we have $\left\|\varrho_{n}+\eta_{n}\right\|_{L_{q}^{v}}=\left\|\varrho_{n}\right\|_{L_{q}^{v}}+\left\|\eta_{n}\right\|_{L_{q}^{v}}$. For $1<q<\infty$, it follows immediately that $\eta_{n}=\varrho_{n}$. For $q=1$, it only follows that the polynomials $\eta_{n}$ and $\varrho_{n}$ have the same sign almost everywhere on $\mathbb{I}$. But the zeros of these polynomials are simple and lie in the interior of the interval $\mathbb{I}$; therefore, the polynomials $\eta_{n}$ and $\varrho_{n}$ have the same set of zeros and, hence, it follows that these polynomials coincide in the case when $q=1$, too.

For a given weight $v$ and a given point $z \in \mathbb{I}$, we define the weight

$$
w(x)=|x-z| v(x)
$$

on the interval $\mathbb{I}$. We denote by $\varrho_{n}^{*}=\varrho_{n, w, q}^{*}$ the polynomial of degree $n \geq 1$ with the unit leading coefficient that deviates the least from zero in the space $L_{q}^{w}=L_{q}^{w}(\mathbb{I})$, i.e., is a solution of the problem

$$
\min \left\{\left\|p_{n}\right\|_{L_{q}^{w}}: p_{n} \in \mathscr{P}_{n}^{1}\right\}=\left\|\varrho_{n}^{*}\right\|_{L_{q}^{w}}
$$

on the set $\mathscr{P}_{n}^{1}$ of polynomials of degree $n$ with the leading coefficient equal to 1 .

The polynomial $\varrho_{n}^{*}$ can be characterized by the property that the function $\left|\varrho_{n}^{*}\right|^{q-1} \operatorname{sign} \varrho_{n}^{*}$ is orthogonal to the space $\mathscr{P}_{n-1}$ (see, for example, [13, Ch. 3, Sect. 3.3, Theorems 3.3.1, 3.3.2]), i.e.,

$$
\int_{\mathbb{I}} w(x) p_{n-1}(x)\left|\varrho_{n}^{*}(x)\right|^{q-1} \operatorname{sign} \varrho_{n}^{*}(x) d x=0, \quad p_{n-1} \in \mathscr{P}_{n-1} .
$$

This property coincides with property (2.9). Therefore, the polynomials $\varrho_{n}$ and $\varrho_{n}^{*}$ differ only by a constant factor. Thus, the following statement holds. 
Corollary 1. Let $z$ be an end point of the interval $\mathbb{I}, 1 \leq q<\infty$, and $n \geq 1$. The polynomial $\varrho_{n}^{*}$ of degree $n$ with the unit leading coefficient that deviates the least from zero in the space $L_{q}^{w}$ with weight (2.11) is the unique extremal polynomial in inequality (2.7).

Special cases of this statement are given in [1, Theorem 1], [2, Theorem 2], [3, Theorem 2], [4, Theorem 3]; they have been proved there by means of other arguments.

\section{Acknowledgements}

The author is grateful to Professor P.A. Borodin for useful and fruitful discussions of the topic of geometry of complex Banach spaces. The author is thankful to the referees who carefully read the paper and made a number of useful suggestions.

\section{REFERENCES}

1. Arestov V.V., Deikalova M.V. Nikol'skii inequality for algebraic polynomials on a multidimensional Euclidean sphere // Proc. Steklov Inst. Math., 2014. Vol. 284. Suppl. 1. P. S9-S23. DOI: $10.1134 /$ S0081543814020023

2. Arestov V., Deikalova M. Nikol'skii inequality between the uniform norm and $L_{q}$-norm with ultraspherical weight of algebraic polynomials on an interval // Comput. Methods Funct. Theory, 2015. Vol. 15, no. 4. P. 689-708. DOI: 10.1007/s40315-015-0134-y

3. Arestov V., Deikalova M. Nikol'skii inequality between the uniform norm and $L_{q}$-norm with Jacobi weight of algebraic polynomials on an interval // Analysis Math., 2016. Vol. 42, no. 2. P. 91-120. DOI: $10.1007 / \mathrm{s} 10476-016-0201-2$

4. Arestov V., Deikalova M., Horváth Á. On Nikol'skii type inequality between the uniform norm and the integral $q$-norm with Laguerre weight of algebraic polynomials on the half-line // J. Approx. Theory, 2017. Vol. 222. P. 40-54. DOI: 10.1016/j.jat.2017.05.005

5. Babenko V.F., Korneichuk N.P., Ligun A.A. Extremal properties of polynomials and splines. New York: Nova Science, 1996.

6. Day M.M. Normed linear space. Berlin; Göttingen; Heidelberg: Springer, 1958.

7. Diestel J. Geometry of Banach spaces: selected topics. Berlin: Springer, 1975.

8. Dunford N., Schwartz J. Linear operators: general theory. New York: Interscience, 1958.

9. Gol'shtein E.G. Duality theory in mathematical programming and its applications. Moscow: Nauka, 1971. 351 p. [in Russian].

10. Handbook of the Geometry of Banach Spaces. Ed. W.B. Johnson and J. Lindenstrauss. Elsevier, 2001. Vol. 1.

11. James R. Characterizations of reflexivity // Studia Mathematica, 1964. Vol. 23, iss. 3. P. 205-216.

12. Kolmogorov A.N., Fomin S.V. Elements of the theory of functions and functional analysis. Moscow: Fizmatlit, 2004.

13. Korneichuk N.P. Extremal problems of approximation theory. Moscow: Nauka, 1976. [in Russian].

14. Milovanović G.V., Mitrinović D.S., Rassias Th.M. Topics in polynomials: extremal problems, inequalities, zeros. Singapore: World Scientific, 1994. 821 p.

15. Nikol'skii S.M. Inequalities for entire functions of finite degree and their application in the theory of differentiable functions of several variables // Trudy Mat. Inst. Steklova, 1951. Vol. 38. P. 244-278 [in Russian].

16. Simonov I.E., Glazyrina P.Yu. Sharp Markov-Nikol'skii inequality with respect to the uniform norm and the integral norm with Chebyshev weight // J. Approx. Theory, 2015. Vol. 192. P. 69-81. DOI: $10.1016 / \mathrm{j}$.jat.2014.10.009

17. Singer I. Best approximation in normed linear spaces by elements of linear subspaces. Berlin: Springer, 1970.

18. Szegő G., Zygmund A. On certain mean values of polynomials // J. Anal. Math., 1953. Vol. 3, no. 1. P. $225-244$. 